\title{
„Wenn ich es nicht tue, dann macht's ein anderer“ - Subjektwerdungen und Verantwortung
}

\author{
Klaus Geiselhart and Tobias Häberer \\ Institut für Geographie, Friedrich-Alexander-Universität Erlangen-Nürnberg, Germany \\ Correspondence: Klaus Geiselhart (klaus.geiselhart@fau.de)
}

Received: 24 August 2018 - Revised: 19 January 2019 - Accepted: 5 March 2019 - Published: 27 March 2019

\begin{abstract}
Kurzfassung. Poststructuralist theory focuses largely on describing how and why subjects reproduce the social conditions they have internalised. This is a deconstruction of the central idea of the Enlightenment, the human capacity for autonomous action. At the same time, however, it also denies all individuals any responsibility and ultimately leads criticism into a crisis. Pragmatist philosophy offers the possibility of determining the role of the mind in processes of becoming a subject without abandoning the achievements of the poststructuralist concept of subjectification. The concept of transaction describes how actors constitute each other as subjects within social situations. The relationships that arise through such processes depend, among other things, on the personalities of those people involved. Accordingly, it is possible to identify the responsibility of individuals to govern their social relations and personality development. Since these aspects can only be determined in localised individual cases, this offers a particularly suitable starting point for geographical critic.
\end{abstract}

\section{Einleitung}

Wer sich heute empirisch mit konkreten Handlungsvollzügen auseinandersetzt, sieht sich mit einer etwas unbefriedigenden Situation konfrontiert. Der Practice Turn hat deutlich gemacht, dass die klassischen Handlungsrationalitäten der Handlungstheorie (zweck-, sinn- und wertrational) allenfalls retrospektive Rationalisierungen erlauben, Handlungen aber niemals in genau diesem Sinne freie, also willentlich intendierte Entscheidungen des Individuums sind. Empirisch kann also allenfalls ein methodologischer Individualismus angesetzt werden (Werlen, 2013). Bei derartiger Forschung ist man sich schon vorab bewusst, dass der eingesetzte handlungstheoretische Blick eine Verkürzung darstellt. In den jüngeren kultur- und sozialwissenschaftlichen Debatten wurde nun vor allem der Begriff der Praktik als konzeptionelle Kern-Kategorie herausgearbeitet (Schmidt, 2012; Hillebrandt, 2014; Reckwitz, 2016). Diese Ansätze fanden auch in der Geographie entsprechende Rezeption (u.a. Everts et al., 2011).

Praktiken sind demnach als konventionalisierte Aktivitätsformen einer Gesellschaft zu denken, welche von den Individuen wiederholt aufgegriffen werden, wodurch sich diese Gesellschaft in ihrer historisch spezifischen Weise reprodu- ziert. Diese Praktiken werden in erster Linie körperlich ausgeführt, entspringen also weitgehend einem praktischen Bewusstsein und sind erst in zweiter Linie mit Verstehensleistungen der Individuen und explizitem Wissen verknüpft. Die meisten praxeologischen Ansätze haben demnach ihre Stärke darin, zu erklären warum Individuen Gesellschaft weitgehend auf bereits etablierte Weise reproduzieren, obwohl vielleicht gesellschaftliche Problematiken ein verändertes Handeln erfordern würden. Nun ist aber auch deutlich, dass es durchaus einen gesellschaftlichen Wandel gibt, doch welche Rolle Individuen bei der Hervorbringung dieses Wandels spielen ist erstaunlich wenig reflektiert. In der Regel werden an dieser Stelle heute poststrukturalistische Theorien eingesetzt und der Wandel bspw. durch Iteration, also durch die unwillentlichen Veränderungen der Praktiken, die in den jeweiligen Einzelausführungen geschehen, erklärt (z.B. Schäfer, 2013). Dies widerspricht aber der Selbstwahrnehmung der Menschen, die uns als Interviewpartner bspw. in der geographischen Stadtforschung begegnen, denn diese verstehen sich durchaus als bewusste, handlungsfähige Subjekte. Eine Reflexion über den Subjektbegriff wird derzeit aber hauptsächlich aus poststrukturalistischer Perspektive geleistet. 
Besonders fruchtbar sind poststrukturalistische Ansätze dahingehend, dass sie Subjektivierungsweisen beschreiben, die aus sozialen Ordnungsstrukturen hervorgehen. Demnach bringt Subjektivierung die Individuen in eine bestimmte gesellschaftliche Position und die Individuen reproduzieren in der Folge dann die Bedingungen ihrer Subjektivierungen. Was diese „Ansätze eint, ist, dass sie alle die Widerständigkeit des Subjekts gegen bestehende hegemoniale gesellschaftliche Verhältnisse betonen" (Dzudzek and Strüver, 2013:147). Dabei bleibt aber unklar, woher genau sich diese Widerständigkeit speist. Dass die gesellschaftliche Praxis trotz aller Kontinuitäten einem Wandel unterliegt wird häufig auf das Konzept der Iterabilität zurückgeführt (Dzudzek, 2013). Widerständigkeit und sozialer Wandel erklären sich entweder aus den Wiederholungen innerhalb der Praxis und den dabei auftretenden Abweichungen, oder auch daraus, dass Subjekte mehrfach konstituiert sind. Die Tatsache, dass sich heute die „Lebensbezüge vervielfacht“ haben, führt dazu, dass das Subjekt die eigene Positionalität, ,immer wieder aufs Neue zu entwerfen hat" (Dzudzek and Strüver, 2013:147). Menschen handeln dann auf unerwartete Weise, wenn in konkreten Situationen verschiedene ihrer Prägungen in Konflikt geraten, sie sozusagen überdeterminiert sind wie bspw. bei einer Lehrerin, die ihr eigenes Kind unterrichten muss. Die Dynamik der Praxis entsteht demnach aus einer Kombinatorik verschiedener strukturell induzierter Prägungen, welche in konkreten Situationen unvereinbar sind wodurch eine Offenheit entsteht, in der sich Performativität entwickeln kann (Dzudzek, 2013; Strüver, 2013). Darüber hinaus betont die poststrukturalistische Position die negativen Auswirkungen einer zunehmenden Selbstverantwortung der Subjekte, in Gegnerschaft zu neoliberalen Tendenzen der Responsibilisierung ${ }^{1}$ (Schwiter, 2013).

Wir möchten in diesem Beitrag aufzeigen, dass ein pragmatistischer Subjektbegriff der poststrukturalistischen Vorstellung von Subjektvierung auf der einen Seite sehr ähnlich ist, durch den veränderten Blickwinkel aber eine Perspektivenerweiterung zulässt, die u.E. insbesondere für geographisches Arbeiten interessant ist. In dieser Perspektive lässt sich Widerständigkeit in einer Dialektik des Selbst verorten, innerhalb derer auch die Rolle des bewussten Denkens, also des Geistes, bestimmt ist. Hierdurch wird persönliche Verantwortung abseits von Responsibilisierung fassbar. Im Folgenden problematisieren wir, dass es Akteure gibt, die ihr Handeln mit Verweis auf die Alternativlosigkeit oder die Unausweichlichkeit bestimmter Entwicklungen mit dem Argument rechtfertigen, dass es immer jemanden geben wird, der diesen Entwicklungen trotz moralischer Bedenken Vorschub

\footnotetext{
${ }^{1}$ Responsibilisierung bezeichnet den gesellschaftlichen Vorgang einer sich verstärkt durchsetzenden Überbetonung persönlicher Verantwortung, die die moralische Vorstellungskraft derart tief erfasst, dass es in vielen gesellschaftlichen Bereichen möglich wird, Verantwortung auf das Individuum abzuwälzen, ohne dass soziale $\mathrm{Ge}$ genwehr erfolgt.
}

leistet, selbst wenn Einzelne sich ihrer erwehren. Derartigen Positionen hat die poststrukturalistische Theorie nichts entgegenzusetzen. Ein Begriff von individueller Verantwortung aber ist u.E. notwendig, um die Angemessenheit menschlichen Handelns beurteilen und entsprechend auch Kritik üben zu können.

\section{Subjektivierung im Poststrukturalismus}

Der Begriff des Subjektes unterlag in den sozial- und kulturwissenschaftlichen Debatten einem enormen Wandel. Klassischerweise wurde das Subjekt mit dem Mentalen in Verbindung gebracht. Bewusstsein wurde als seine Basis und Notwendigkeit angesehen (Frank, 1988; Reckwitz, 2008). Demnach braucht der Mensch den Geist, um ein Subjekt sein zu können und nur mittels des Geistes ist er fähig nicht nur die Welt, sondern auch sich selbst zu erkennen. Im kartesianischen Sinne wird das Subjekt bspw. verstanden als etwas, das aller Erfahrung zugrunde liegt, eine Instanz, die dem Denken vorausgeht, die einen festen Bezugspunkt darstellt (Volbers, 2017). Prototypisch steht dafür auch die Descartsche Philosophie, die den Geist zur eigenständigen Instanz, der res cogitans, erhob, indem sie ihn vom Materiellen unterschied. In der Selbstvergewisserung des ,ich denke also bin ich“ erkennt sich demnach das Subjekt selbst als Gegenstück zur Objekthaftigkeit der ihm äußeren Welt.

Diese neuzeitliche Erfindung des Subjekts wurde nun aber vom Poststrukturalismus vehement in Frage gestellt. Geist ist demnach keineswegs die zentrale Instanz des Subjekts. Da das Subjekt ebenso von Effekten der Körperlichkeit abhängig ist, wird das poststrukturalistische Subjekt häufig als dezentriert angesehen. Subjektivität wird als das Ergebnis psycho-physischer Prozesse verstanden. Michel Foucault, Jaques Lacan, Judith Butler und Ernesto Laclau haben distinkte, subjektorientierte Forschungsheuristiken entwickelt, denen, so betont Reckwitz (2008), gemeinsam sei, dass sie die Frage darauf richteten, wie sich Individuen unbewusst den kulturellen Ordnungen ihrer Gemeinschaften unterwerfen. Subjektivität entsteht innerhalb der gesellschaftlichen Ordnung, wobei Identität eine zentrale Komponente des Subjekts ist. Identität wird dabei verstanden als ,die Identifizierung der einzelnen Person als Wesen mit bestimmten Eigenschaften in Differenz zu anderen im Rahmen der kulturellen Subjektordnung" (Reckwitz, 2008:79). Im poststrukturalistischen Denken werden Subjektpositionen demnach als Identitäten repräsentiert. Dies geschieht, weil Individuen sich zum Zwecke der Selbstvergewisserung und Selbstbeschreibung an in diskursiven Kontexten fixierten Vorstellungen orientieren und diese sich ihnen, auch vermittelt durch die gesellschaftliche Praxis, körperlich einschreiben. Aber auch Identitäten sind dezentriert. Da sie zu ihrer Bestimmung ein $\mathrm{Au}-$ ßen benötigen, von dem sie sich differenztheoretisch unterscheiden, sind sie auch auf diese Weise in Diskurse eingeschrieben. Identitäten repräsentieren soziale Positionen, die 
vermittelt durch Diskurse als Macht wirken (auch: MachtWissen), durch die die entsprechenden Subjekte erst hervorgebracht werden. So beschreibt Michel Foucaults Konzept der Subjektivierung, wie Individuen mittels sogenannter Technologien des Selbst versuchen, den an ihre Identitätskategorie gestellten Anforderungen gerecht zu werden (Foucault, 2009[1982/83]). Der Poststrukturalismus etabliert damit eine Gegenposition zu Vorstellungen, die den Menschen als souveränes, eigenständig denkendes und handelndes Wesen ansehen. Die Vorstellung einer Human Agency und das Empfinden von Selbstwirksamkeit sind demnach Illusionen. Der Mensch wird primär als ein durch Prägung und Anpassung geformtes Wesen angesehen. Dies bedeutet nicht weniger als die Dekonstruktion der Vorstellung des Menschen als autonomen Schöpfer seiner Selbst.

Möchte man aber nicht nur die beständige Reproduktion bestehender gesellschaftlicher Institutionen erklären, sondern auch den empirisch sehr wohl beobachtbaren Wandel etablierter Rollenbilder oder auch das Zustandekommen gesellschaftlichen Widerstands, dann bedarf es weiterführender Überlegungen. So erhält das Subjekt einen Funken von Autonomie, ,wenn es denn wahr ist, daß es keinen anderen, ersten und letzten Punkt des Widerstands gegen die politische Macht gibt als die Beziehung seiner selbst zu sich." (Foucault, 2016[1981/82]:313). Die Technologien des Selbst seien Foucault zufolge derjenige ,Typus von Praktiken [...], denen er zuspricht, sich - zumindest potenziell und auch nur partiell und momenthaft - aus der Umklammerung des Macht-Wissens zu lösen“ (Münte-Goussar, 2015:120). Gouvernemental präfigurierte Subjektivierungen könnten hierbei durch Wahl von geeigneten Techniken sozusagen subvertiert werden. Es bleibt jedoch fraglich, inwieweit diese marginale Fähigkeit zu steuerndem Handeln wirklich von Foucault angedacht wurde oder, ob sie erst in der Rezeption hineingelesen wurde, weil sich hier eine offene Flanke in der Theoriebildung auftut, die nach Klärung verlangt. In dem für Foucault charakteristischen historischen Stil konzentriert er sich weitergehend darauf, $\mathrm{zu}$ beschreiben, wie $\mathrm{zu}$ verschiedenen Zeiten verschiedene Umgangsweisen mit dem eigenen Selbst dem Menschen als heilbringend galten, sodass ,er einen gewissen Zustand des Glücks, der Reinheit, der Weisheit, der Vollkommenheit oder der Unsterblichkeit erlangt“ (Foucault, 1993[1982]:26).

In der Regel entstehen nonkonforme Effekte bei der Subjektpositionierung im poststrukturalistischen Denken nicht als autonome Leistung des Individuums, sondern auf Grundlage einer Kombinatorik. Wenn in einer Situation mehrere widerstreitende Subjektivierungen eines Individuums gleichzeitig zum Tragen kommen, dann entsteht eine Überdeterminierung und damit die Möglichkeit neuer Modi des Handelns. Weil keine der etablierten Verhaltensweisen angewendet werden kann, ohne mit anderen etablierten Verhaltensweisen in Konflikt zu geraten, ist Handeln in diesem Moment prinzipiell offen. Aufgrund dieser Möglichkeit, eine mehrfache Ausprägung des Ichs zu denken, könne Foucault auch als ein „späterer Bruder im Geiste“ John Deweys angesehen werden (Keller, 2014:73). „Ein Individuum als ein Mitglied verschiedener Gruppen kann in sich selbst geteilt sein und im wahren Sinne widerstreitende Ichs besitzen oder ein vergleichsweise desintegriertes Individuum sein“ (Dewey, 1996[1927]:160). Dieser Aspekt tritt auch beim Subjektivierungskonzept Judith Butlers hervor. So schreibt sie in Hinblick auf geschlechterspezifische Identitäten, ,that multiple and coexisting identifications produce conflicts, convergences, and innovative dissonances within gender configurations which contest the fixity of masculine and feminine placements with respect to the paternal law" (Butler, 1999:85 f.). Laut Butler (1997) zeigt sich auch der iterativ-performative Charakter von Praktiken als Faktor des Wandels. Da in der Praxis keine Situation der anderen identisch ist, muss sich der Vollzug relevanter Praktiken immer an einem spezifischen Setting ausrichten. Subjektivierung zeigt sich demnach auch in der Kompetenz, Praktiken entsprechend zu justieren. Dadurch ist jeder Ausführung auch die Möglichkeit der Abweichung inhärent. Butler beschreibt damit eine ,iterability of the subject that shows how agency may well consist in opposing and transforming the social terms by which it is spawned" (Butler, 1997:29). Derartige Konzeptionen werden mitunter kritisch kommentiert: „Die poststrukturalistische [sic] Perspektive Judith Butlers arbeitet sich an dem philosophischen Problem ab, die Freiheit eines Handlungsvermögens zu begründen, das - so die Annahme - durch Determination und Unterwerfung hergestellt wird. Das mag für Ansätze einer kritischen Soziologie interessant sein, die sich von den Theorietraditionen Frankfurter Provenienz absetzen will. Sie tut dies jedoch um den Preis des erneuten Versuches, die ,Eigentlichkeit des Subjekts' zu begründen, also Subjektphilosophie nach der Subjektphilosophie zu betreiben“" (Keller, 2014:81).

Die Stärke der poststrukturalistischen Subjektkonzeption liegt in der kritischen Analyse etablierter gesellschaftlicher Subjektivierungsweisen. Diese Analysen können sozialen Bewegungen, wie etwa der Genderbewegung, als Basis der Argumentation dienen. Die Erklärung aber, wie und warum es zu sozialen Bewegungen kommt, warum Menschen trotz ihrer Prägungen gegen etablierte Verfahrensweise aufbegehren, ist und bleibt eine Herausforderung für die poststrukturalistische Theoriebildung. Aber der Poststrukturalismus ist nicht die einzige Theorietradition, die eine rein intentionalistisch gedachte Vorstellung eines autonomen, rationalen Subjekts in Frage stellt. Eine Verortung des Subjektes in verkörperten Verhaltensweisen, sedimentierten Überzeugungen, Gewohnheiten und/oder Routinen wird ebenfalls in vielen praxisorientierten Ansätzen vorgenommen. Die Frage aber, welche Rolle der Geist, also bewusstes, reflektierendes Denken, bei der Formierung körper-geistiger Subjektwerdungen spielt, wird u. E. lediglich vom klassischen Pragmatismus explizit behandelt. 
3 Die pragmatistische Perspektive auf Subjektwerdungen als situative Ereignisse

Das Denken des klassischen Pragmatismus lässt sich ebenfalls in die subjektkritische Tradition einordnen (Volbers, 2017). Um aber die etwas andere Stoßrichtung verstehen zu können, ist es notwendig einen Schritt zurücktreten und die Begriffe Individuum, Subjekt, Identität und Selbst zu differenzieren. Dabei werden wir auch auf die Begriffe der Person und der Persönlichkeit treffen und sehen, wie all diese Begriffe eine Sozialität voraussetzen, die oftmals vernachlässigt wird. „Eine Person, ein Selbst, ein Subjekt zu sein sind Funktionen, die sich aus komplex organisierten organischen und sozialen Interaktionen ergeben“ (Dewey, 1995[1929]:205). Ähnlich der poststrukturalistischen Kritik weist John Dewey darauf hin, dass der Individualismus des modernen Lebens dazu neigt, den Menschen aus dem gemeinschaftlichen Leben herauszuabstrahieren, wodurch das Subjekt als moderne Erfindung etabliert wurde. „In jedem Falle ist ein Individuum nicht länger einfach etwas Partikulares, ein Teil, der seine Bedeutung ausschließlich in einem Ganzen hat, sondern ein Subjekt, ein Selbst, ein charakteristisches Zentrum von Begehren, Denken und Hoffen“ (Dewey, 1995[1929]:212-213).

Dewey macht deutlich, wie der Geist mittels der Anschauung eine Vorstellung von der Welt, bestehend aus ihm äußerlichen Objekten, erschafft. Weil diese Konstruktionsleistung individuell vorgenommen wird, geschähe dies ,,in einem Medium, das ganz eigentlich subjektiv genannt werden kann“ (Dewey, 1995[1929]:216). Vernachlässigt werde dabei jedoch häufig, dass derartige Erschaffungen auf Kommunikation, auf gemeinschaftliche Sinnproduktion angewiesen und ausgerichtet sind. Nach Georg Herbert Mead (1998[1934]) kann ein Individuum nur Bewusstsein von etwas erlangen, indem es sich fragt, was wohl die anderen Mitglieder seiner Gemeinschaft über diese Sache sagen würden. Mead beschreibt das als eine Hereinnahme des Gesellschaftlichen in das Individuum. Ebenso verhält es sich mit der Vorstellung vom eigenen Selbst, also der eigenen Identität. Diese kann nur mittels einer Antizipation der Meinungen anderer exploriert werden. Die Aufklärung und die Moderne seit der Renaissance hätten hingegen, so Dewey, das Erkennen des eigenen Selbst in einer Weise befördert, die zur Selbst-Isolierung des Ichs geführt hätten und blind machten für diese Eingebundenheit dieses Ichs in den gesellschaftlichen Austausch. Seine beispiellose Attraktivität beziehe dieser Subjektivismus dabei aus der vom Individuum empfundenen konstruktiven Macht des eigenen individuellen Denkens. In solitärer Kontemplation, also dann, wenn man alleine im Studierzimmer denkt, ist der Geist fähig die größtmögliche Illusion von Klarheit zu erzeugen. Die Bedingungen des Lebens erscheinen nun durchschaubar und damit zumindest potentiell auch gestaltbar zu sein. Dem Individuum verschlossen bleibt dabei, dass dies nur möglich ist, weil relativierende, auf Komplexität hinweisende Irritationen ausgeblendet werden. Ein derartiger Subjektivismus ist damit der ständige Begleiter ei- nes Objektivismus, der durchaus begründet bestimmte Kategorien entwirft und diese fortan für existent oder zumindest für geklärt ansieht. Damit erhebt sich das denkende Individuum mittels seiner rational kontemplativen Fähigkeiten über das eigene situative Dasein hinaus. Hat das Individuum aber auf diese Weise eine vermeintlich weitreichende Klarheit erzeugt, so kommt es früher oder später nicht umhin, das eigene, denkende, fühlende und handelnde Selbst in den von ihm selbst geschaffenen Kategorien zu erklären und damit sein eigenes singuläres Erleben seiner eigenen Konstruktion Untertan zu machen. An dieser Stelle zeigen sich in der Regel dann auch die Grenzen der entworfenen Erklärungsleistungen.

Das Individuum besitzt demnach eine „Zweideutige Natur des Selbst“, es nimmt eine „doppelte Stellung“ ein, zwischen den Erklärungsleistungen seines Egos und dem Erleben einer äußeren, als gegeben erscheinenden Welt der Dinge und Personen (Dewey, 1995[1929]:237). Das Individuum erlebt sich selbst in einer beständigen Spannung zwischen dem, was es als objektiv vorhanden annimmt und der $\mathrm{Ab}$ schätzung dessen, was es an diesen Bedingungen zu erklären oder sogar zu verändern vermag. In seinem Erleben ist es gespalten zwischen der Notwendigkeit Bedingungen akzeptieren zu müssen, die unhintergehbar zu sein scheinen und der vagen Idee, es gäbe vielleicht doch eine Möglichkeit die äußeren Bedingungen zu verändern. Diese doppelte Bewegung etabliert die Bewusstheit eines Menschen in einem beständigen Alternieren zwischen einem Konstruieren und einem Erleben von Welt, also zwischen der geistigen Erschaffung von Objekten und dem praktischen Erleben der Relevanz dieser geschaffenen Kategorien (vgl. zu diesen Ausführungen auch Volbers, 2017). Demzufolge ist es dem Einzelnen ,gleichermaßen natürlich wie unvermeidlich, [auch] sein eigenes Selbst innerhalb geschlossener Grenzen zu definieren und dann zu versuchen, das Selbst in expansiven Akten zu erproben, die unvermeidlich zuletzt in einem Zusammenbruch des eingemauerten Selbst enden. Hier liegt die letzte ,Dialektik' des Allgemeinen und des Individuellen“ (Dewey, 1995[1929]:236-237), die sich in einer beständig neu justierten Spannung zwischen gesellschaftlicher Anpassung und Selbstbehauptung äußert. Dies stellt sich als ein psychophysischer Prozess der gelebten Erfahrung dar. Der Mensch als Körper-Geist-Wesen (Body-Mind) befindet sich in einem fortlaufenden Lernprozess der beständigen Auseinandersetzung mit den Bedingungen der Welt. Dabei stehen geistige Aktivitäten in einer beständigen Rückbindung an praktische Tätigkeiten und es finden emotionale Bewertungen statt, die die jeweils weitere Geneigtheit des Individuums beeinflussen. Die Fähigkeit des Menschen Erfahrungen zu machen charakterisiert, so Dewey, den Menschen als Körper-GeistWesen.

Erfahrung ist damit aber nichts Individuelles. Zwar sind es einzelne Menschen, die Erfahrungen machen, doch erfahren sie die Bedingungen ihrer Welt und ihre Gemeinschaft. Mead (1998[1934]) beschreibt wie Individuen zwi- 
schen zwei Modi des Erlebens des eigenen Selbst alternieren. Auf der einen Seite konstruieren sie ein „,me“ indem sie ihre eigene Identität auf gesellschaftlichen Rollenerwartungen und Identitätskategorien aufbauen. Auf der anderen Seite erleben sie eine letztlich wenig kontrollierbare Instanz ihres eigenen Selbst, das „I“, welches immer ein Stück weit mit den Rollenerwartungen der Identitätskonstruktionen in Konflikt gerät. Auf der einen Seite erfahren Individuen Gemeinschaft, ganz konkret in Traditionen, Bräuchen, Praktiken und Institutionen, wodurch dafür nützliche Kompetenzen und ein Verständnis gesellschaftlicher Rollen erschaffen werden. Individuen werden in einer bestimmten Gesellschaft erfahren, wenn sie lernen, geschickt und sozial kompetent zu handeln. Sie entwickeln ein Gespür dafür, was sie von anderen erwarten können, wenn sie selbst sich auf die in dieser Gemeinschaft üblichen Arten und Weisen verhalten. Aber die Menschen machen ihre Erfahrungen auf einzigartige Weise. Sie erleben sich selbst einerseits als charakteristischen Teil ihrer Gemeinschaft, gleichzeitig aber auch als Ausnahme. Sie erleben sich als typisches Mitglied und gleichzeitig auch als Singularität. Sie übernehmen entsprechend ihrer sozialen Identität konventionalisierte Positionen, wie z.B. als Kinder, Erwachsene, Schüler, Lehrer, Väter, Mütter, Berufstätige oder religiöse Personen, doch gleichzeitig stehen sie mit diesen gesellschaftlichen Positionen in Konflikt.

Der Mensch wird erfahren, indem er lernt in einer Gesellschaft kompetent zu handeln. Das ist dem ähnlich, was der Poststrukturalismus als Subjektivierung beschreibt. Erfahrung ist also weitgehend ein Erlernen und Begreifen gesellschaftlicher Bedingungen, aber darüber hinaus sind nach pragmatistischer Vorstellung die Individuen aktiv und kreativ an diesen Prozessen beteiligt. Dem Individuum begegnen innerhalb der konkreten Situationen des ErfahrungenMachens bestimmte emotionale Qualitäten, anhand derer es sich seiner selbst gewahr wird. „Unterhalb und innerhalb dieser Geschehnisse, nicht außerhalb von ihnen oder ihnen zugrundeliegend, finden sich jene Ereignisse, die Selbste genannt werden“ (Dewey, 1995[1929]:226-227). Bruchstückhaft nur erscheint dem Individuum die Wahrnehmung einer Situation und seines Eingebundensein in diese Situation, innerhalb der es sich auch seines eigenen Verhaltens bewusst wird. Man erlebt sich selbst nur selten als souverän, häufig eher als re-agierend denn als agierend, man könnte auch sagen getrieben. Im Eifer des Gefechtes ist eine rationale Steuerung des eigenen Verhaltens kaum durchgängig möglich, vielmehr reagiert man meist auf Basis individueller, erworbener und angeborener Dispositionen. Diese Dispositionen geben den einzelnen Individuen ihr jeweils charakteristisches Verhalten, welches auch als deren Persönlichkeit bezeichnet wird (vgl. auch Volbers, 2017). Im pragmatistischen Denken bildet also nicht Identität die wesentliche subjektrelevante Kategorie. Inwieweit jemand in identitätsrelevanten Kategorien, bspw. Geschlecht, Beruf, soziale Funktion etc., angesprochen wird, ist situationsabhängig. Was aber über alle Situationen hinweg als weitgehend konstant be- schrieben werden kann, ist die Art und Weise, wie ein bestimmter Mensch auf Bedingungen, die ihm begegnen, reagiert. Die Persönlichkeitspsychologie befasst sich eben mit dieser Tatsache, dass Individuen nicht immer gleich oder vorhersehbar reagieren, aber aufgrund eines charakteristischen Verhaltens trotzdem als eine bestimmte Person identifiziert werden können (Asendorpf und Neyer, 2012). Die Persönlichkeit macht einen Menschen irgendwie berechenbar, aber keineswegs kann aus der Persönlichkeit eines Menschen all ihr Handeln abgeleitet werden. Persönlichkeit ist demnach ein sozial sehr wirkungsvoller Einfluss, trotzdem erweist sich der Begriff als gesellschaftstheoretisch weitgehend vernachlässigt.

\section{Persönlichkeit als vernachlässigte gesellschaftstheoretische Kategorie}

Betrachtet man Kategorien der Persönlichkeitspsychologie, dann wird deutlich, dass es dabei immer um Charakteristika geht, die das soziale Verhalten von Personen betreffen (Asendorpf und Neyer, 2012). Die fünf Dimensionen der Persönlichkeit, die im psychologischen Jargon auch häufig als „big five" bezeichnet werden, sagen primär etwas über den Umgang eines Menschen mit sich selbst und Anderen aus. Menschen sind in unterschiedlichem Maße offen für neue Erfahrungen. Sie unterscheiden sich in ihrer Gewissenhaftigkeit, also darin wie genau oder perfekt sie ihre Aufgaben erfüllen wollen. Sie treten unterschiedlich zurückhaltend oder offensiv auf, unterscheiden sich also in ihrer Extraversion. Ihre soziale Kompetenz lässt sie unterschiedlich emphatisch, kooperativ und rücksichtsvoll sein und schließlich sind Individuen in unterschiedlichem Maße emotional verletzlich. Menschen denen Persönlichkeitsstörungen der sogenannten ,dunklen Triade“, Narzissmus, Machiavellismus oder Psychopathologie, attestiert werden, zeichnen sich vor allem durch unsoziale, manipulative oder herrschsüchtige Verhaltensweisen aus oder gar dem Fehlen jeglicher Empathie.

Im Angesicht der Tatsache wie elementar Persönlichkeiten das soziale Miteinander beeinflussen, erscheint die mangelnde gesellschaftstheoretische Auseinandersetzung mit dem Thema geradezu fahrlässig. Man mache es sich vielfach zu einfach, so Manfred Lutz (2009), wenn man, wie es häufig geschehe, bspw. Adolf Hitler als psychisch krank bezeichnet, denn dieser hätte in unserer Gesellschaft niemals die für eine Diagnose notwendigen Bedingungen erfüllt. Er hätte sich selbst niemals als leidend präsentiert und nach Maßgabe seiner ganz eigenen Vorstellung, war er wohl durchaus erfolgreich. So behandele unsere Gesellschaft die Falschen, nämlich die, die am Wohle der Gemeinschaft interessiert wären, und die, die an den Schwierigkeiten des sozialen Miteinanders verzweifelten. Jene aber, die sich wenig um das Wohl anderer kümmern, haben hingegen oftmals einen Vorteil. So kann sich eine narzisstische Persönlichkeitsstörung, die sich ja vor allem durch manipulatives Verhalten auszeich- 
net, in vielen Fällen sogar karrierebegünstigend auswirken (vgl. auch Schneck, 2018). Die mangelnde Auseinandersetzung der Gesellschaftstheorie mit dem Begriff der Persönlichkeit ist auch nicht nachvollziehbar, wenn man bedenkt, dass es in der Praxis vielfach üblich ist, z.B. in Vorstellungsgesprächen, gezielt Persönlichkeitsmarker, wie Teamfähigkeit, Offenheit etc. abzufragen. Distinktion anhand von Persönlichkeitsmerkmalen wird also durchaus praktiziert, theoretisch aber kaum reflektiert.

Diese Zurückhaltung der Gesellschaftstheorie bezüglich einer Betrachtung von Persönlichkeit ist wahrscheinlich auf die Nähe des Begriffes zu dem der Person zurückzuführen. Der Begriff Person markiert den Unterschied zwischen dem Status ein Jemand und nicht nur ein Etwas zu sein (Spaemann, 1996). Eine Person zu sein setzt die Anerkennung in einer Gemeinschaft voraus. Diese humanistische Idee war Grundlage der Etablierung von Persönlichkeitsrechten und damit auch der Formulierung der Menschenrechte (Joas, 2015). Persönlichkeitsrechte erheben denjenigen, dem der Status als Person zugestanden wird, gegenüber demjenigen, der diesen Status nicht bekommt, wie das bspw. historisch beim Umgang mit Sklaven der Fall war (Lotter, 2012). Ebenso zeigt der Begriff der persona non grata, dass es geschehen kann, dass eine Person in einer Gemeinschaft nicht mehr erwünscht ist. Es kann also auch Gründe geben, einem Menschen den Status einer Person zu entziehen. Interessanterweise unterliegt das, was als eine Person angesehen wird, in hohem Maße der kulturellen Prägung (Geertz, 1987:294). Gesellschaften entwickeln demnach spezifische Vorstellungen, unter welchen Bedingungen die vollständig entfaltete Würde einer Person auch vollständige Persönlichkeitsrechte beinhalten sollte. Auseinandersetzungen mit dem Begriff der Person laufen demnach Gefahr in Verdacht zu geraten, ob sie denn auch wirklich alle Menschen gleichbedeutend als Personen ansähen oder, ob sie nicht insgeheim Kriterien für bessere und schlechtere Menschen formulieren (Spaemann, 1996; Lotter, 2012). Diese Diskussionen können hier nicht ausgeführt werden, doch in Anbetracht der Erkenntnis, dass die Persönlichkeitsentwicklung eines Menschen nicht nur genetisch, sondern immer auch sozial bedingt ist (Asendorpf und Neyer, 2012:261ff.), wird die Dringlichkeit deutlich, Fragen nach den Bedingungen gelingender Persönlichkeitsentwicklung und den Möglichkeiten der selbstgesteuerten Entwicklung der eigenen Persönlichkeit zu stellen.

\section{Transaktion, Persönlichkeiten und Beziehungen}

Persönlichkeit sagt also nichts darüber aus, was jemand tut, sondern vielmehr, wie jemand in sozialen Situationen für gewöhnlich reagiert. Das Wissen um die Persönlichkeit eines Menschen hilft, dessen Verhalten zu antizipieren. Es lässt uns abschätzen, ob sich die Person in einer bevorstehenden Situation tendenziell aufbrausend, leicht reizbar, leicht verletzlich, aggressiv oder eher zuvorkommend und hilfsbereit verhal- ten wird. Persönlichkeit beeinflusst nicht nur das Verhalten der Person selbst, sondern auch das Verhalten anderer gegenüber dieser Person. Persönlichkeit zeigt sich nur, wenn eine soziale Situation eintritt, also wenn ein Individuum in Kontakt mit Dingen oder anderen Menschen affiziert wird. Das Verhalten eines Individuums ist durch seine Persönlichkeit aber keinesfalls determiniert, vielmehr stellt die Persönlichkeit eine Disposition dar, mittels derer Individuen den gesellschaftlichen Positionen, die sie einnehmen, ein individuelles Gesicht geben. Die Kenntnis der charakteristischen Verhaltenstendenzen einer Person kann nun von anderen Akteuren genutzt werden, um in der Beziehung zu dieser Person erwünschte Ergebnisse zu erzielen. Dabei werden Normen und Grenzen zwischenmenschlichen Verhaltens beständig situativ gezogen und erlebt.

Menschen erfüllen ihre Rollen, Aufgaben und Pflichten in einer für sie charakteristischen Weise. Sie geben ihren gesellschaftlichen Positionen ein einzigartiges Gesicht, sie werden also nur vermittelt durch ihre Persönlichkeiten zu Subjekten und das tun sie auch niemals alleine, sondern immer nur in der Begegnung mit einem sozialen Gegenüber in konkreten Situationen. Im Moment des Aufeinandertreffens machen sich Menschen gegenseitig zu Subjekten, es findet Transaktion statt. Im Gegensatz zum Konzept der Interaktion, das von bereits existierenden festen Entitäten ausgeht, bezeichnet Transaktion die Tatsache, dass sich die Beteiligten in einer Situation gegenseitig hervorbringen (Dewey und Bentley, 1991[1949]; Steiner 2014). Menschen und andere Objekte werden nur im Kontakt zueinander zu dem, als was sie wahrgenommen werden. Sie fixieren sich gegenseitig auf eine Möglichkeit ihrer Selbst, die erst in dieser Situation räumlich und zeitlich konkret werden kann. Somit können Aspekte wie bspw. das räumliche Setting einer konkreten Situation oder die Konstellation der beteiligten Akteure in den Fokus der Betrachtung rücken. In der gegenseitigen Hervorbringung als Subjekte bildet sich eine Beziehung zwischen den beteiligten Personen heraus, die so nur durch und zwischen exakt diesen Individuen existieren kann (Saunders, 2005). Persönlichkeiten haben maßgeblich Einfluss darauf, wie Individuen in Situationen zu Subjekten werden und damit auch darauf, welchen Charakter die Beziehungen zwischen ihnen annehmen. Das Vorhandensein eines Chefs und eines ihm unterstellten Teams garantiert noch lange kein produktives Arbeiten. Es ist von enormer Bedeutung wie sich die Beziehungen zwischen den Beteiligten ausprägen. Die Beziehungen wiederum entwickeln Pfadabhängigkeiten, die ein gemeinsames Handeln auch langfristig entweder erleichtern oder, im Falle „belasteter Beziehungen“, verkomplizieren.

\section{Subjekt und Geist}

Ein Subjekt kann man also nur sein, wenn man sozial auf jemand anderen oder etwas anderes bezogen ist. Ein Subjekt zu sein bedeutet affiziert zu sein, was wiederum heißt, dass 
man nur in sozialen Konstellationen und nur in Situationen, oder allgemeiner in auf Situationen bezogenem Denken, zum Subjekt wird. Ein Subjekt zu werden bedeutet eine Beziehung mit anderen zu leben, sozial angesprochen zu werden, aktiviert zu werden und durch die eigenen Reaktionen wiederum andere zu aktivieren. Es bedeutet nicht nur eine gesellschaftliche Position einzunehmen, sondern auch betroffen zu sein und Haltungen zu entwickeln. Im Gegensatz zur Selbstidentität, die sich weitgehend aus intellektueller Reflexion über das eigene Selbst speist, muss Subjektivität praktisch vollzogen werden. Subjektwerdung beinhaltet reaktives Verhalten. Jeder kennt die Erfahrung, dass man sich im Nachhinein über das eigene Verhalten ärgert. Nicht immer erscheint die eigene Art und Weise zu reagieren im Nachhinein effektiv oder angemessen. Aber genau dieses Erleben von Getriebenheit, dieses Ausgeliefertsein eröffnet im pragmatistischen Denken ein Fenster für Selbstwirksamkeit: eine Möglichkeit der Einflussnahme des Geistes.

Individuen handeln in der Regel auf der Grundlage eines ,System[s] von Überzeugungen, Kenntnissen und Unkenntnissen, von Zustimmung und Ablehnung, von Erwartung und Würdigung von Bedeutungen, die unter dem Einfluß von Brauch und Tradition eingeführt worden sind" (Dewey, 1995[1929]:215). Individuen, die mit Verstand ausgestattet sind, meistern demnach ihren Alltag weitgehend, indem sie ihrem Sinn für das Gemeinsame (Common Sense) folgen. Hier zeigt sich, dass Geist kein rein individuelles Phänomen ist, sondern, dass dieser nur auf der Ebene ,der Vergemeinschaftung, der Kommunikation und Teilhabe" hervorgebracht wird (Dewey, 1995[1925]:261; vgl. auch Volbers, 2017). Zwar ist es das einzelne Individuum das denkt, doch reproduziert es in weiten Teilen seines Denkens lediglich intellektuelle Konventionen seiner Gemeinschaft. Das Selbst wird sich dieses Kontextes seines Handelns normalerweise nicht bewusst, zumindest solange dieser nicht brüchig wird, bspw. wenn Vorhaben nicht wie üblich ablaufen oder die erworbenen Fähigkeiten nicht weiterhelfen. In solchen Situationen, zwischen den Gewohnheiten und Routinen, beginnen Individuen nachzudenken. Mit dem Impuls zum Nachdenken über bisherige Selbstverständlichkeiten erhalten die Individuen die Chance, wie Dewey sagt, ihren Geist zu individualisieren, also die Chance über die in ihrer Gemeinschaft konventionalisierten Grenzen hinauszudenken. Und es sind derart individuelle Geister, die die Voraussetzung für eine kritische Position bilden. Deweys (1995[1925]:214) Unterscheidung zwischen mit Geist ausgestatteten Individuen und individuellen Geistern bestimmt demnach die menschliche Wirkmächtigkeit als eine Fähigkeit zur Reflexion über die eigenen Ansichten, Verhaltensweisen und gesellschaftlichen Prägungen.

Der Pragmatismus bestimmt demzufolge weder ein autonomes Subjekt im Sinne der Aufklärung, noch ein völlig dezentriertes, jeglicher Handlungsfähigkeit beraubtes Subjekt (Abb. 1). Er skizziert ein situativ reaktives Emergieren von Subjekten, das in gewisser Weise ,riskant“ genannt werden

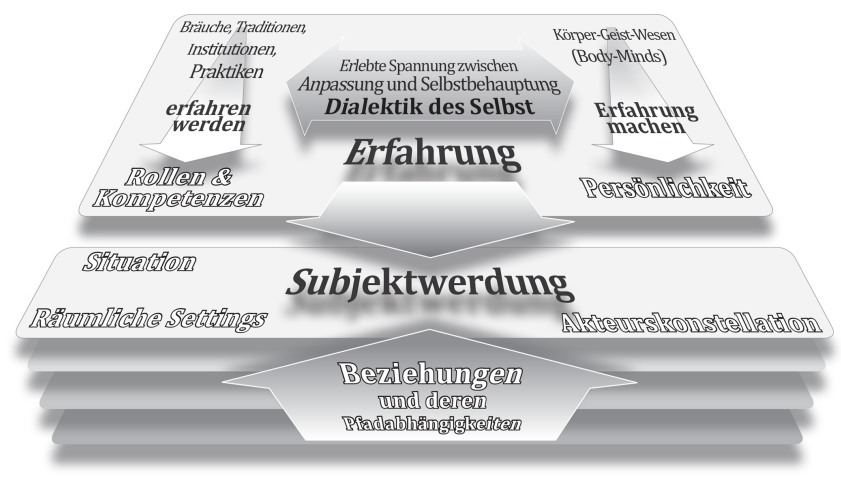

Abb. 1. Subjektwerdung als situatives Ereignis.

kann, weil es immer auf impliziten, verkörperlichten sozialen Dispositionen aufbaut, die nicht immer auch erwünschte soziale Resonanzen bewirken (Volbers, 2017). Darüber hinaus bestimmt der Pragmatismus eine Fähigkeit des Individuums zur Reflexion über die eigenen Dispositionen derartiger Subjektwerdungen. Das Individuum kann seine eigenen Subjektwerdungen zwar nicht direkt und unmittelbar beherrschen, doch kann es danach streben seine Dispositionen zur Subjektwerdung zumindest langfristig in einem gewünschten Sinne zu verändern. Der Pragmatismus bestimmt also die Human Agency als Fähigkeit, die eigene Prägung zu hinterfragen und die Voraussetzungen des eigenen Verhaltens $\mathrm{zu}$ korrigieren. Dies kann auch als Aufforderung zur Persönlichkeitsentwicklung gelesen werden.

\section{Auf der Suche nach der Verantwortung}

Das poststrukturalistische Subjekt wird von den gesellschaftlichen Verhältnissen hervorgebracht, denn sowohl seine Überzeugungen wie auch seine Wünsche sind von diesen bestimmt. Letztlich ist das poststrukturalistische Individuum damit auch jeglicher Verantwortung enthoben. Es subjektiviert sich entsprechend der produktiven Macht, ohne einen eigenen selbstbestimmten Anteil daran zu haben. Aber nach derartigem Subjektverständnis können allenfalls abstrakte Instanzen wie Kapitalismus, Rassismus oder Sexismus als Ursachen von Ungleichheiten und Ungerechtigkeiten benannt werden. Sicherlich ist es wichtig der Kritik derartige abstrakte Kategorien zur Verfügung zu stellen, doch kann derart abstrakte Kritik nur dann transformative Kraft entwickeln, wenn im tatsächlichen gesellschaftlichen Leben jemand die Transferleistung vollbringt und diese abstrakten Kategorien in konkreten Ereignissen auf bestimmte konkrete Akteure bezieht und diesen Verantwortung zuschreibt. Unterlässt man eine Reflexion der Bedingungen unter denen derartige Zuschreibungen geschehen sollten, dann riskiert man zweierlei.

Erstens kommt es nicht selten vor, dass dabei Grenzen der Angemessenheit überschritten werden (Edlinger, 2015; Weiss, 2017). Um nur wenige Beispiele zu nennen stellte 
sich die Frage der Angemessenheit bspw. als beim Kölner NoBorderCamp im Juli 2012 Aktivisten der Critical Whiteness Bewegung das Recht einforderten, Redebeiträge zu stoppen, wenn sie persönlich diese als rassistisch empfinden (ak, 2013), oder als in den USA eine jüdische Lesbe von einer Homosexuellenveranstaltung verwiesen wurde, weil sie ein T-Shirt mit der Aufschrift ,Proud Jewish Dyke“ trug. In der Vorstellungswelt einiger von der Intersektionalitätsdebatte inspirierten TeilnehmerInnen passte das nicht zu der Auffassung des Zionismus als Täterkategorie (Weiss, 2017).

Zweitens birgt das Versäumnis zu klären, unter welchen Bedingungen Kritik geübt werden sollte, die Gefahr, dass Muster der Kritik missbraucht werden. So werden bspw. heute nicht selten feministische Ansprüche auf Selbstbestimmung und Fragen der Sicherheit von Frauen vor Übergriffen zur Rechtfertigung einer anti-islamischen, fremdenfeindlichen Gesinnung herangezogen (Delfin, 2016). Entsprechend attestiert Bruno Latour (2007[2004]:16), dass die Waffen der Kritik ,über unklar gezogene Grenzen geschmuggelt wurden und der falschen Partei in die Hände gerieten“. Nun will sich Latour nicht ,einfach damit beruhigen, daß bad guys nun einmal jede Waffe benutzen, derer sie habhaft werden können“ (2007[2004]:11), sondern fragt danach, ob nicht bei der Formulierung der Waffen der Kritik etwas vergessen wurde. In diesem Geiste wollen wir im Folgenden einer Haltung nachspüren, die u.E. gezielt Verantwortung leugnet und damit letztlich auch Ausdruck einer hauptsächlich am eigenen Vorteil interessierten Persönlichkeit ist. Wenn zur Begründung dieser Haltung aber poststrukturalistische Theorie herangezogen wird, was leicht möglich ist, weil diese in diesem Bereich eine Leerstelle aufweist, dann wird der Poststrukturalismus zum unfreiwilligen Erfüllungsgehilfen.

Die weit verbreitete Haltung des ,wenn ich es nicht mache, dann macht"s ein anderer" drückt die Rechtfertigung aus, etwas trotz besseren Wissens zu tun. Bei dieser Haltung geht es nicht darum ein einmaliges Ausnahmeverhalten zu rechtfertigen. Einmaliges unnachhaltiges, umweltschädigendes, konsumistisches oder anderweitig abträgliches Verhalten kann auf vielfältige Weise entschuldigt werden, bspw. mit den gegenwärtigen Umständen, dem Fehlen von Alternativen oder auch dem aktuellen Wunsch nach Bequemlichkeit oder Genuss. Derartiges Verhalten würde im Falle einer Unterlassung auch nicht von einer anderen Person ausgeführt werden. Mit Verweis auf eine gesamtgesellschaftliche Dimension würden diesbezüglich eher Entschuldigungen wie ,ich wäre ja blöd, wenn ich es nicht mache, denn alle anderen tun es ja auch" angewendet. Hingegen weist aber die Äußerung „wenn ich es nicht mache, dann macht's ein anderer" unmissverständlich darauf hin, dass die sich derart äußernde Person vor hat eine gesellschaftlich relevante Handlung auszuführen oder eine Position einzunehmen, die anderenfalls von einer anderen Person übernommen werden würde. Zudem ist es eine Formel, die die mit dieser Position verbundenen gesellschaftlichen Verantwortung bewusst nicht thematisiert.
Es geht hier aber keineswegs darum, Verantwortung ausschließlich an den Folgen individuellen Handelns festzumachen. Auf der einen Seite ist es wünschenswert, wenn jeder Einzelne sich im Sinne einer Verantwortungsethik (z.B. Jonas, 1979) unsozialer oder umweltschädlicher Verhaltensweisen bewusst wird und diese zu vermeiden sucht, aber eine direkte Verantwortung für die ökologischen und sozialen Probleme unserer Zeit kann der Einzelne nicht übernehmen. Diese zu lösen muss vielmehr als eine politische Aufgabe angesehen werden. Leider habe sich, so Yascha Mounk (2017), eine Rhetorik der persönlichen Verantwortung nicht nur in Ratgeberliteratur, Kolumnen und der politischen Sprache durchgesetzt, sondern auch unsere moralische Vorstellungskraft tief erfasst und sogar die Natur der Wohlfahrtsstaaten verändert. Das Individuum wird in einem MaBe verantwortlich gemacht, die es Kraft seiner individuellen Wirksamkeit und der Zukunftsoffenheit des eigenen Lebens und der gesellschaftlichen Entwicklungen gar nicht übernehmen kann. Es hat eine Naturalisierung von Verantwortung und deren Verkörperungen in den Subjektvierungsweisen der Individuen stattgefunden. Diese Tendenz wird von poststrukturalistischen Autoren zu Recht kritisiert, streben sie doch eine Entunterwerfung der Subjekte von derartigen moralischen Imperativen an.

Die von den Beförderern derartiger Responsibilisierung erhobenen Argumente entsprechen aber ironischerweise durchaus der poststrukturalistischen Logik. Bestimmte Verhaltensdispositionen einer Bevölkerungsmehrheit, so wird argumentiert, müssen ökonomisch oder politisch als Voraussetzung betrachtet werden (subjektivierende Macht). Solange sich dieser Diskurs nicht von der Basis her verschiebe, könne man auch nichts ändern, ohne existenzgefährdende Verluste an Wählerstimmen oder Kunden in Kauf nehmen zu müssen. Die Formel „,wenn ich es nicht tue, dann macht's ein anderer" ist nun genau der Ausdruck dieser Haltung, die mit Hinweis auf die strukturellen Gegebenheiten verschleiert, dass der Sprecher sich genau diese Gegebenheiten zum Vorteil macht. Die Formel behauptet, dass es im Prinzip egal sei, ob nun der Sprecher oder eine andere Person von der Übernahme der von ihm eingenommenen Position profitiere, weil das für diejenigen, die von den von dieser Stelle ausgehenden Wirkungen betroffen sind oder eventuell sogar geschädigten werden, letztlich keinen Unterschied mache.

Verschwiegen wird in der Regel, dass mit Übernahme der zur Debatte stehenden gesellschaftlichen Position Verantwortung verbunden ist, derer sich die sprechende Person bewusst stellen sollte, wenn sie diese Position übernimmt. Die Formel verschweigt, dass man mit Macht ausgestattete Positionen besser oder schlechter, autoritärer oder sozialer sowie demokratischer oder autokratischer ausfüllen kann. Sie formuliert bewusst nicht, wie sie gedenkt diese Position auszufüllen oder warum sie glaubt für diese Position besonders geeignet zu sein. Sie verschweigt, dass mit Übernahme einer gesellschaftlich einflussreichen Position, auch ein Anspruch an die Persönlichkeit des diese Position überneh- 
menden Menschen gestellt wird. Es ist diese Zurückweisung von Verantwortung mit Verweis auf die übermächtige gesellschaftliche Struktur, die ganz auf Linie mit den poststrukturalistischen Vorstellungen von Subjektivierung liegt.

\section{Die Verantwortung zur Persönlichkeitsentwicklung}

Aus pragmatistischem Verständnis aber kann eine andere Art von Verantwortung benannt werden. Diese stellt keine überhistorische oder über alle gesellschaftlichen Bedingungen hinweg gegebene Konstante dar. In demokratischen Gesellschaften aber ist eine gesellschaftliche Position mit der Verantwortung verbunden, alle Menschen im Einflussbereich dieser Position gleichermaßen als Personen anzusehen. Die Frage nach der „Person“ ist letztlich die Frage nach der Anerkennung eines lebendigen Wesens als ein Individuum mit Rechten in einer Gemeinschaft gleichartiger Wesen. Die Stärke des Begriffes „Person“ liegt aber nicht in seiner definitorischen Kraft zur Normsetzung, sondern vielmehr im Potenzial, eine Basis für Kommunikation in gegenseitiger Anerkennung darzustellen.

Die Frage nach der Person ist letztlich auch die Frage danach, was man glaubt von anderen Menschen erwarten zu können (Lotter, 2012:13). Im alltäglichen empathischen Miteinander unter Menschen entfalten sich Erwartungen wie auch Verantwortungsbewusstsein immer situativ. Entscheidungen darüber, was richtiges oder falsches Handeln ist, ergeben sich nach Maßgabe einer empfundenen Angemessenheit, die sich an den Erfordernissen der gelebten sozialen Beziehungen, der gesellschaftlichen Aufgaben und Rollen orientiert. In diesem Sinne sind soziale Kompetenzen, also die intuitiven und emotionalen Fähigkeiten wichtiger, als die kognitiven Fähigkeiten zum rationalen Denken, für das in gegebenen Situationen naturgemäß die Zeit und der Überblick fehlt. „Daher ist die Ausbildung der Fähigkeit, Konkretes differenziert zu erfassen, von unverzichtbarer und in vielen Hinsichten primärer moralischer Bedeutung. Eine Person kann die Überzeugung hegen, anderen Menschen in Schwierigkeiten nach Möglichkeit helfen zu sollen (und dies auch auf postkonventionelle Weise formulieren), ohne je in eine solche Gelegenheit zu kommen, weil sie die psychische und soziale Lage ihrer Mitmenschen schlichtweg nicht wahrnimmt. Auch bei voller Entwicklung der logischen Fähigkeiten kann es ihr an der dafür erforderlichen Sensibilität, Lebenserfahrung und Empathie fehlen" (Lotter, 2012:347).

Verantwortliches Handeln erfüllt sich demnach nicht in theoretischen Grundsätzen oder Handlungsanweisungen, sondern in der empathischen Fähigkeit abstrakte Grundsätze situationsbezogen angemessen anzuwenden, also in einer sozial integrativen Persönlichkeit, die ihre Handlungsspielräume intuitiv gemeinwohlorientiert anwenden kann. Dazu muss ein Individuum zur sozialen Inter- bzw. Transaktion fähig sein, also die Fähigkeit besitzen in sozialen Begegnungen verantwortungsbewusst zum Subjekt zu werden. Verantwortlichkeit erwächst demnach aus der menschlichen Fähigkeit zur Reflexion über das eigene Handeln und der damit verbundenen Möglichkeit die eigene Persönlichkeit zu bilden. Persönlichkeitsentwicklung wird damit zur Aufgabe, sich verantwortungsvoll in die Gesellschaft einzupassen.

Dies entspricht auch einem weitverbreiteten Gerechtigkeitsempfinden, das letztlich auch in unserem Justizsystem abgebildet ist. Demnach ist es nicht gerecht, würde sich Schuld und das Strafmaß nur am Tatbestand des Vergehens bemessen. Vielmehr erscheint es angemessen vor Festsetzung des Strafmaßes zu klären, ob eine Person überhaupt schuldfähig ist, also mental ausreichend zur Selbstreflexion fähig ist und, ob ein Bewusstsein für die Schuld und entsprechend Reue vorhanden sind. Wir gehen davon aus, dass Menschen prinzipiell dazu in der Lage sind, sich ihren Prägungen zu entziehen. So kann bspw. eine schlechte Kindheit strafmildernd wirken, wird aber nicht bedingungslos als Entschuldigung für jedes Fehlverhalten akzeptiert. Um sich seinen Prägungen zu entziehen, muss man sein Denken individualisieren, man muss sich darauf einlassen, Erfahrungen $z u$ machen. Man muss es zulassen, sich zu verändern und sich der eigenen Zukunftsoffenheit stellen. „Das alte Selbst wird abgelegt, das neue Selbst bildet sich erst noch“ (Dewey, 1980[1934]:238, vgl. auch 35ff.).

\section{Fazit: Konsequenzen für die Kritik}

Die pragmatistische Kritik verschiebt den Fokus von Identität auf Beziehung. Nicht immer begegnen Menschen einander im Modus etablierter Differenzkategorien. So ist nicht jeder männliche Chef seiner weiblichen Angestellten gegenüber ein Patriarch. Vielmehr bauen beide Beteiligten ihre Praxis auf den derzeitigen Bedingungen auf, in denen Genderungerechtigkeiten leider immer noch vorhanden sind. Nun kann ein Unternehmer aber entweder diese Ungerechtigkeiten für seine Vorteile ausnutzen oder er kann versuchen diese zumindest für seinen Betrieb zu minimieren. Dabei sind nicht nur Identitätskategorien relevant, denn es spielen vielfältige Fragen eine Rolle (betriebliche Altersvorsorge, Arbeitsbedingungen, Lohngleichheit, etc.). Ebenso sind auch Fälle individueller Fürsorge interessant, bspw. wenn ein Unternehmer einen bestimmten Angestellten bereitwillig weiterträgt, auch in einer längeren Phase verminderter Leistungsfähigkeit, z.B. ausgelöst durch eine persönliche Krise oder Krankheit. Dem Unternehmer muss zweifellos zugestanden werden, bei derartigen Maßnahmen nur so weit zu gehen, wie er glaubt im Wettbewerb mit anderen Unternehmen, die für ihre Mitarbeiter nicht im selben Maße soziale Bedingungen schaffen, noch konkurrenzfähig bleiben zu können. Verantwortung liegt demnach in der angemessenen Berücksichtigung seiner eigenen und der Bedürfnisse anderer.

Durchaus komplizierter gestalten sich derartige Überlegungen, wenn man an Führungspositionen innerhalb größe- 
rer Firmenzusammenhänge denkt, deren Befugnisse deutlich eingeschränkter sein dürften, als die eines selbstständigen Unternehmers. Führungskräften, die bei der Umsetzung von auf höherer Ebene beschlossenen Firmenstrategien moralische Bedenken haben, bleibt gefühlt oftmals nur die Anpassung, sofern unklar ist, ob es der Kariere schaden würde, die Beschlüsse in Frage zu stellen. Eine Rechtfertigung im Sinne des ,wenn ich es nicht tue, dann tut es ein anderer“ liegt dann berechtigter Weise sehr nahe.

An dieser Stelle öffnet sich eine bisher weitgehend unerkundete Dimension der Gesellschaftskritik. Dazu zählen Fragen, wie: $\mathrm{Zu}$ welchen Persönlichkeiten müssen Menschen in den verschiedenen gesellschaftlichen Bereichen werden, um dort bestehen zu können? Welche Status können nur unter Aufgabe bestimmter gemeinschaftorientierter Haltungen erreicht werden? Für welche gesellschaftliche Positionen sollten bestimmte Persönlichkeitsmerkmale wünschenswerterweise ausgeprägt sein? Oder mittels welcher Strategien verhindern es Unternehmen, dass Verantwortung eindeutig festgemacht werden kann?

Die Stärke pragmatistischen Denkens ist es aber, dass es in Anerkennung der Dialektik des Selbst nicht nur in die Richtung des Allgemeinen führt, sondern ebenfalls in Richtung des singulären Einzelfalles. Die pragmatistische Kritik ist auch eine Kritik der Mediation, die die Zwänge der Verhältnisse anerkennt und neben einer oppositionellen Kritik der strukturellen Verhältnisse auch nach den im Einzelfall jeweils zugrunde liegenden Voraussetzungen fragt. Sie denkt den Begriff Macht lokalisiert und konkretisiert, um zu sehen, wie sich Macht in der Komplexität der Praxis gebrochen zeigt. Einerseits zu zeigen, wie Macht-Wissen die Menschen durchdringt und sie letztlich der Fähigkeit, Verantwortung zu übernehmen, beraubt, andererseits aber auch aufzuzeigen, wie im lokalisierten Geschehen bestimmte Einzelpersonen oder Interessenverbände über ein notwendiges $\mathrm{Ma} ß$ hinaus diese Macht zu ihrem persönlichen Gewinn ausnutzen. Diese Kritik stellt die Fragen der Verhältnismäßigkeit und ist damit zwangsläufig geographisch, denn nur in der Konkretheit des lokalen Kontextes lassen sich derartige Verantwortlichkeiten identifizieren und entsprechende Vorwürfe konkretisieren.

Es ist eine Frage der Persönlichkeit, ob jemand bestehende Ungleichheiten für seine Zwecke ausnutzt oder nicht. So weiß jeder männliche Chef um die gendertheoretische Kritik, doch weiß er ebenso um geeignete Strategien der Rechtfertigung (Boltanski und Thévenot, 2007). Letztlich ist es von seiner persönlichen Haltung abhängig, ob er seinen Entscheidungsspielraum nutzt diese Ungerechtigkeiten zu minimieren oder nicht. Es ist eine Frage der Persönlichkeit, inwieweit eine Person die Augen vor gesellschaftlichen Spannungen verschließt oder ob sie sich Missstände bewusst macht, ob sie Ambiguitäten der Praxis aushalten kann und welche persönlichen Konsequenzen sie daraus zieht.

Die Persönlichkeitspsychologie beschäftigt sich damit, zu ergründen, warum Menschen trotz aller Konformität in ihrem Verhalten doch als einzigartig erkennbar sind. Menschen ha- ben keine Subjektivität in gleicher Weise wie sie eine Persönlichkeit besitzen. Subjektivität bildet sich in jeder einzelnen Situation erneut aus, doch unsere Persönlichkeit beeinflusst, wie wir in Situationen reagieren, ob wir bspw. tendenziell eher konfrontativ oder vermittelnd eingreifen. Unsere Persönlichkeit ist es, über die wir Kontrolle erlangen müssen, wenn wir nicht wieder und wieder auf die gleiche Art und Weise reagieren wollen. Der Pragmatismus formuliert ein reflexives Individuum, das transaktionalen, situativen Subjektwerdungen unterliegt, auf Basis derer es die individualisierende Funktion seines Geistes aktivieren kann. In kontinuierlichen Erfahrungsprozessen kann es gelingen über die eigenen gesellschaftlichen Prägungen und Selbstverständlichkeiten hinauszuwachsen. Dies stellt sich langfristig als ein Prozess der Persönlichkeitsentwicklung dar, im Sinne eines bewussten Umgangs mit den eigenen als negativ empfundenen Persönlichkeitsmerkmalen. Aber Persönlichkeitsentwicklung wäre unvollständig ohne eine Reflexion über den Sinn des gesellschaftlichen Daseins, also ohne ein Nachdenken über die Frage, was Gemeinschaft bedeutet, in welchem Maße der Mensch und damit man selbst auf Gemeinschaft angewiesen ist, wo die Grenzen von Vergemeinschaftungsprozessen naturgemäß verlaufen, also auch die Grenzen sozial akzeptablen Verhaltens.

Diese Ansprüche müssen letztlich aber auch an die Arbeit des Wissenschaftlers angelegt werden. Laut Luc Boltanski und Eve Chaipello (2003:524) erwächst Kritik aus Empörung. Empörung erwächst aber nicht aus unserer gesellschaftlichen Position als Wissenschaftler. Unsere Persönlichkeit ist es, die die Schwelle bestimmt, ab der wir uns empören, die uns gleichzeitig aber auch auf einen Weg bringt, mit dieser Empörung umzugehen. Das bedeutet: nur, wenn wir uns selbst als Persönlichkeit ernst nehmen, dann können wir auch als Wissenschaftler Kritik üben und unterliegen nicht den Launen unseres Selbst. Wir müssen Verantwortung dafür übernehmen, dass wir, wie alle anderen Menschen auch, manchmal geneigt sind, willentlich wegzusehen, aber ebenso dafür, dass wir oftmals die Tendenz haben, uns blindlings zu empören. Wir können Verantwortung erst dann konkret zuschreiben, wenn wir den Ursprung unserer Empörung, der naturgemäß ein spezifisches Ereignis war, genau betrachtet haben.

Es scheint als habe der Hang der Soziologie, ihre Begriffe immer gesamtgesellschaftlich zu denken, dazu geführt, dass die „pragmatische Wende“ der Kritik (Celikates, 2009:27) sich letztlich doch wieder in abstrakten Kategorien einer Soziologie der Kritik (Bogusz, 2010; Peter, 2011) oder denen einer Theorie der Praxis verlor (siehe hierzu Boltanskis Kritik der Bourdieuschen Kritik, 2010:40ff.). Die geographische Kritik kann aber ihren Blick auf konkrete Beziehungen richten, um bei den konkreten Vorgängen der Praxis zu bleiben. Damit wird Kritik zwangsweise lokal, sie muss lokale Entwicklungspfade nachzeichnen, um dann konkrete Geschehnisse, Entscheidungen oder auch Einzelpersonen für entstandenes Unrecht verantwortlich machen zu können. Es wird 
immer Menschen oder Gruppen von Menschen geben, die Kraft ihrer gesellschaftlichen Stellung zu ihrem persönlichen Nutzen und zum Nachteil anderer handeln. Auch die entpersonalisierte poststrukturalistische Macht wird im lokalen Kontext letztlich von konkreten Personen oder Personengruppen ausgeübt. Dabei ist es von entscheidender Bedeutung, inwieweit Fragen des Gemeinwohls Berücksichtigung finden. Wenn sich die Kritik zutraut in derartigen Fällen Verantwortung zuzuschreiben, kann sie u.E. transformative Kraft entfalten.

Datenverfügbarkeit. Für diesen Artikel wurden keine Datensätze genutzt.

Interessenkonflikt. Die Autoren erklären, dass kein Interessenkonflikt besteht.

Begutachtung. This paper was edited by Benedikt Korf and reviewed by two anonymous referees.

\section{Literatur}

ak: Dimensionen der Differenz, in: ak, Analyse \& Kritik, Zeitung für linke Debatte und Praxis, 584, 21 June 2013, online aufrufbar: http://www.akweb.de/ak_s/ak584/51_web.htm (letzter Zugriff: 22 March 2019), 2013.

Asendorpf, J. B. und Neyer, F. J.: Psychologie der Persönlichkeit, Springer, Berlin, 2012.

Bogusz, T.: Zur Aktualität von Luc Boltanski, VS Verlag, Wiesbaden, 2010.

Boltanski, L.: Soziologie und Sozialkritik: Frankfurter AdornoVorlesungen 2008, Suhrkamp, Frankfurt a.M., 2010.

Boltanski, L. und Chiapello, É.: Der neue Geist des Kapitalismus, UVK Verlagsgesellschaft, Konstanz, 2003.

Boltanski, L. und Thévenot, L.: Über die Rechtfertigung. Eine Soziologie der kritischen Urteilskraft, HIS, Hamburg, 2007.

Butler, J.: The Psyche of Power: Theories in Subjection, Stanford University Press, Stanford, 1997.

Butler, J.: Gender Trouble: Feminism and the Subversion of Identity, Routledge, New York und London, 1999.

Celikates, R.: Kritik als soziale Praxis: Gesellschaftliche Selbstverständigung und kritische Theorie, Campus, Frankfurt a.M., 2009.

Delfin, R.: Former Miss Belgium Anke Van dermeersch: Islam Threatens Women's Rights And Muslim Immigration Must Be Stopped, in: Critical Beauty, online aufrufbar: http://www.criticalbeauty.com/2016/05/ former-miss-belgium-anke-van-dermeersch.html (letzter Zugriff: 22 March 2019), 2016.

Dewey, J.: Kunst als Erfahrung, Suhrkamp, Frankfurt a.M., 1980[1934].

Dewey, J.: Erfahrung und Natur, Suhrkamp, Frankfurt a.M., 1995[1929].

Dewey, J.: Die Öffentlichkeit und ihre Probleme, Wissenschaftliche Buchgesellschaft, Darmstadt, 1996[1927].
Dewey, J. und Bentley, A.: Knowing and the known, in: Later works 1925-1953, vol. 16, Herausgeber: Boydson, J. A., Southern Illinois University, Carbondale, 97-209, 1991[1949].

Dzudzek, I.: Unternehmen oder Unvernehmen? - Über die Krise des Kreativsubjekts und darüber hinaus, Geogr. Helv., 68, 181-189, https://doi.org/10.5194/gh-68-181-2013, 2013.

Dzudzek, I. and Strüver, A.: Editorial Subjectivities in crisis, Geogr. Helv., 68, 145-152, https://doi.org/10.5194/gh-68-145-2013, 2013.

Edlinger, T.: Der wunde Punkt. Vom Unbehagen an der Kritik, Suhrkamp, Berlin, 2015.

Everts, J., Lahr-Kurten, M., und Watson, M.: Practice matters! Geographical inquiry and theories of practice, Erdkunde, 65, 323334, 2011.

Frank, M.: Subjekt, Person, Individuum, in: Die Frage nach dem Subjekt, Herausgeber: Frank, M., Raufet, G., und van Reijen, W. Suhrkamp, Frankfurt a.M., 1988.

Foucault, M.: Technologien des Selbst, in: Technologien des Selbst, Herausgeber: Martin, L. H., Gutman, H., und Patrick H. H., S. Fischer Verlag, Frankfurt a.M., 24-62, 1993[1982].

Foucault, M.: Die Regierung des Selbst und der anderen: Vorlesung am Collège de France 1982/83, Suhrkamp, Frankfurt a.M., 2009[1982/83].

Foucault, M.: Hermeneutik des Subjekts: Vorlesung am Collège de France 1981/82, Suhrkamp, Frankfurt a.M., 2016[1981/82].

Geertz, C.: Dichte Beschreibung: Beiträge zum Verstehen kultureller Systeme, Suhrkamp, Frankfurt a.M., 1987.

Hillebrandt, F.: Soziologische Praxistheorien, eine Einführung, Springer VS, Wiesbaden, 2014.

Joas, H.: Sind die Menschenrechte westlich?, Kösel, München, 2015.

Jonas, H.: Das Prinzip Verantwortung. Versuch einer Ethik für die technologische Zivilisation, Insel Verlag, Frankfurt a.M., 1979.

Keller, R.: Assoziationen: Über Subjektprobleme des Poststrukturalismus und die Perspektive der Wissenssoziologischen Diskursanalyse, in: Wer oder was handelt? Zum Subjektverständnis der hermeneutischen Wissenssoziologie, Herausgeber: Poferl, A und Schröer, N., Springer VS, Wiesbaden, 67-94, 2014.

Latour, B.: Elend der Kritik: Vom Krieg um Fakten zu Dingen von Belang, Diaphanes, Zürich, 2007[2004].

Lotter, M.-S.: Scham, Schuld, Verantwortung: Über die kulturellen Grundlagen der Moral, Suhrkamp, Frankfurt a.M., 2012.

Lutz, M.: Irre - Wir behandeln die Falschen: Unser Problem sind die Normalen - Eine heitere Seelenkunde, Gütersloh, Penguin, 2009.

Mead, G. H.: Geist, Identität und Gesellschaft, Suhrkamp, Frankfurt a.M., 1998[1934].

Mounk, Y.: The age of responsibility. Luck, Choice, and the Welfare State, Harvard University Press, London, 2017.

Münte-Goussar, S.: Dispositiv - Technologien des Selbst - Portfolio, in: Medien - Bildung - Dispositive: Beiträge zu einer interdisziplinären Medienbildungsforschung, Herausgeber: Othmer, J. und Weich, A., Springer VS, Paderborn, 109-127, 2015.

Peter, L.: Soziologie der Kritik oder Sozialkritik? Zum Werk Luc Boltanskis und dessen deutscher Rezeption, in: lendemains 36, 73-89, online aufrufbar: http://www.periodicals.narr. de/index.php/Lendemains/article/download/203/187 (letzter Zugriff: 22 March 2019), 2011. 
Reckwitz, A.: Subjekt/Identität: Die Produktion und Subversion des Individuums, in: Poststrukturalistische Sozialwissenschaften, Herausgeber: Moebius, S. und Reckwitz, A., Suhrkamp, Frankfurt a.M., 75-92, 2008.

Reckwitz, A.: Kreativität und soziale Praxis, Studien zur Sozialund Gesellschaftstheorie, transcript, Bielefeld, 2016.

Saunders, H. H.: Politics is about relationship, Palgrave, New York, 2005.

Schäfer, H.: Die Instabilität der Praxis, Reproduktion und Transformation des Sozialen in der Praxistheorie, Velbrück Wissenschaft, Weilerswist, 2013.

Schmidt, R.: Soziologie der Praktiken, konzeptionelle Studien und empirische Analysen, Suhrkamp, Berlin, 2012.

Schneck, C.: Coaching und Narzissmus, Wiesbaden, Springer, 2018.

Schwiter, K.: Neoliberal subjectivity - difference, free choice and individualised responsibility in the life plans of young adults in Switzerland, Geogr. Helv., 68, 153-159, https://doi.org/10.5194/gh-68-153-2013, 2013.
Spaemann, R.: Personen. Versuche über den Unterschied zwischen ,etwas“ und ,,jemand“, Klett-Cotta, Stuttgart, 1996.

Steiner, C.: Von Interaktion zu Transaktion - Konsequenzen eines pragmatischen Mensch-Umwelt-Verständnisses für eine Geographie der Mitwelt, Geogr. Helv., 69, 171-181, https://doi.org/10.5194/gh-69-171-2014, 2014.

Strüver, A.: „Ich war lange illegal hier, aber jetzt hat mich die Grenze übertreten,, - Subjektivierungsprozesse transnational mobiler Haushaltshilfen, Geogr. Helv., 68, 191-200, https://doi.org/10.5194/gh-68-191-2013, 2013.

Volbers, J.: Subjektivierung der Erfahrung. Zu Deweys Rekonstruktion der Subjektivität, in: John Dewey: Erfahrung und Natur, Herausgeber: Hampe, M., de Gruyter, Berlin, 97-112, 2017.

Weiss, V.: Die autoritäre Revolte. Die Neue Rechte und der Untergang des Abendlandes, Klett-Cotta, Stuttgart, 2017.

Werlen, B.: Gesellschaft und Raum: Gesellschaftliche Raumverhältnisse Grundlagen und Perspektiven einer sozialwissenschaftlichen Geographie, EWE, 24, 3-16, 2013. 